\title{
A Novel User and Antenna Selection Techniques in Massive MIMO 5G Wireless Communication System
}

\author{
Tasher Ali SHEIKH, Joyatri BORA, Md. Anwar HUSSAIN \\ Dept. of Electronics and Communication Engineering, North Eastern Regional Institute of Science and Technology, \\ Nirjuli, Arunachal Pradesh 791109, India \\ tasher.ece@gmail.com
}

Submitted January 29, 2020 / Accepted June 23, 2020

\begin{abstract}
In this paper, we have proposed a new paradigm for user scheduling in large-scale multiple-input multipleoutput (MIMO) time division duplexing (TDD) system. In this paper, we have selected the users from different groups with semi-orthogonal (SO) and random criterion. We separate the users in different groups with the K-means clustering algorithm which assigns the users into different groups. After user groups are so determined, we use two new user selection paradigms where users are selected in two methods - firstly we select the users in intra-group those are SO with each other along with it also SO with other groups' users. Secondly, users are selected from inter-group those are SO with each other also SO with other group users. In both the selection schemes antennas are scheduled based on the maximum gain of the channel. In the results, it is noticed that in intra-group with semiorthogonal user selection (SUS) and antenna selection (AS) using the zero-forcing $(Z F)$ precoding shown the highest systems rate. We also evaluated the computation cost of our modified proposed algorithm which is exposed in table1. We explored the efficiency of the proposed schemes through MATLAB simulations.
\end{abstract}

\section{Keywords}

Intra-group, inter-group, massive MIMO, user selection, antenna scheduling, complexity

\section{Introduction}

The high data-rates have an ever-increasing requirement from the last few years in the wireless communication network. Few advanced technologies have been proposed like multiple-input multiple-output (MIMO) with time division duplex (TDD), frequency division duplex (FDD), orthogonal frequency division multiplexing (OFDM), nonorthogonal multiple access (OFDM) to meet this demand. But the difference between the exponential growth of demand and supply of data-rates in wireless communication system is always increasing. For this requirement a new technology is looked for in wireless communication network. An advanced technology so-called large-scale
MIMO or massive MIMO has been proposed in [1] to provide services for this demand. This technology has the advantage of significantly increasing the system sum-rate by adding large number of antennas at the base station (BS) to simultaneously serve huge number of users. This emerging technology has many benefits such as system robustness, higher throughput, lower power consumption, simple signal processing technique, simplified multiple access layer. Additionally, it significantly enhanced the system sum-rate and minimized the computational cost [2-4]. A lab demo about the advantages of massive MIMO is also exposed in [5], [6].

It is observed that in massive MIMO network huge number of antennas are deployed at the transmitter and receiver sides. These give the system more degrees of freedom, resulting in higher reliability and system capacity. By simultaneously serving to many users, massive MIMO might be a suitable technology for providing higher system data-rate and throughput. But, to achieve the channel state information (CSI) at the transmitter side is a very difficult task in these systems. Therefore, it is a challenging job in massive MIMO to simultaneously serve the huge number of users [4]. The TDD mode [7-10] is widely considered in all the existing works on massive MIMO. Since, the downlink channel may estimate by exploiting channel reciprocity through the uplink training. This mechanism is also called as a green technology as it consumes minimum power. Unfortunately, there is no such manifest in FDD systems, rather it consumed huge power and spectrum. Since, it required separate pilot-based channel estimation and uplink channel feedback. Recently, in [11] a two-stage precoding scheme has been proposed to reduce the CSI feedback and pilot overhead in the FDD system.

Based on the same two-stage precoding framework, authors in [12] proposed a design of user grouping and scheduling of users in massive MIMO FDD system. In [13] a new agglomerative clustering method is proposed to efficiently simplify the user clustering process. In paper [14] the authors have developed three novel similarity measures algorithms for user grouping such as weighted likelihood, subspace projection and Fubini-study method. They also suggested two clustering scheme, i.e. hierarchical and $K$-means clustering. In [15], a dynamic user 
clustering algorithm is proposed which does not require covariance matrix estimation; hence it reduces the channel feedback. To improve the throughput and fairness of the system a joint user grouping and resource allocation scheme with dual-layer beamforming TDD-long term evolution-Advance (LTE-A) system is proposed in [16].

For multi-user MIMO downlink system, a joint spatial division and multiplexing (JSDM) were explored in [17]. A random-based dynamic grouping strategy is proposed in [18] for cooperative coevolution to cope with large scale multi-objective optimization problems. A joint grouping and scheduling scheme are proposed in [19] for fair user scheduling in large scale MIMO network for the application of internet of things scenario. Based on users' classification in [20], a low complexity two-stage user scheduling scheme is proposed. [21] proposed a cooperative user scheduling algorithm in massive MIMO system specifically to reduce the number of feedback users and decrease the system computational complexity.

From the above literature survey, it is observed that there are no such schemes which jointly applied the user grouping, user selection, and antenna scheduling algorithm with low complexity in massive MIMO to improve the system data-rate. We use four methods jointly like user grouping, user selection, antenna scheduling and precoding scheme in massive MIMO system for data-rate enhancement. In general, to achieve the higher system data-rate and to reduce computational complexity, an optimal grouping method and determining the best users in each group is most important. To resolve this issue, in this paper, we considered $K$-means clustering method for user grouping. For user selection we consider semi-orthogonal user selection (SUS) and random user selection (RUS). If there are only a few active users, some groups may barely have users while some other groups are overloaded. This work will motivate the researchers who are working on massive MIMO for system date-rate improvement. Since, we jointly use user grouping, user selection, and antenna scheduling with precoding schemes for enhancement of system datarate in massive MIMO. follows:

The contribution of the paper is summarized as

- Grouping the users with $K$-means clustering algorithm.

- This work proposes two user selection methods, such as intra-group and inter-group SUS algorithm, intragroup and inter-group RUS algorithm.

- Antennas are scheduled based on the maximum gain of the channel.

- We use ZF and minimum means square error (MMSE) precoding schemes for reducing the interference amongst its own group and other group users which results in improving the system data-rate.

- The calculated computational complexity of modified SUS proposed algorithm is explored in Tab. 1.

The rest of the paper is presented as follows. In Sec. 2, the system model is described in detail. The algo- rithm is defined in Sec. 3. Simulation results are discussed in Sec. 4 and finally concluded with a conclusion in Sec. 5.

Notations: Throughout the paper, unless otherwise specified, bold lower case, bold upper case and upper-case Greek letters are used to represent vectors, matrices, and sets, respectively. Correspondingly $\mathbb{C N}(0, \mu)$ represents the complex Gaussian distribution.

\section{System Model}

A downlink TDD based massive MIMO network is considered for our analysis. We considered the BS has $M$ antennas and $N$ users having both single transmission and reception antenna. The model is shown in Fig. 1. In Fig. 1 we have marked an area as a weak signaling zone [22] because the BS signals strength is very feeble and users on that area generally very negligible. Hence, we considered that area's radius is $R_{\mathrm{w}}=120 \mathrm{~m}$. Excluding the weak zone, the users are distributed uniformly and that area's radius is $R=1000 \mathrm{~m}$. We have divided the $N$ number of users into $G=6$ i.e. $G=\left\{g_{1}, \ldots, g_{6}\right\}$ a number of groups, using the $K$ means clustering method. Such that $N=G U$, where $U$ is the number of users consisting in each group. During the transmission and reception of data at the transmitter and receiver scheduled and selected $u=24$ numbers of antennas and user pairs from $M$ antennas and $N$ users respectively for each time. We also considered that the channel is small scale fading (SSF) characteristics and large-scale fading (LSF), and the channel coefficients are Rayleigh fading in nature. The downlink $N \times 1$ received signal vector $\mathbf{r}$ is given by (1)

$$
\mathbf{r}=\sqrt{\rho} \mathbf{Q} \mathbf{x}+\mathbf{z}
$$

where $\rho$ and $\mathbf{z}$ are the scalar downlink transmit power and additive white Gaussian noise. The $\mathbf{x} \in \mathbb{C}^{M \times 1}$ is the transmit data signal vector having power constraint $\mathrm{E}\left\{\|\mathbf{x}\|^{2}\right\}=1$, where $\mathbf{x}=\mathbf{P} s$ with $s=\left[\mathrm{s}_{1}, \mathrm{~s}_{2}, \ldots, \mathrm{s}_{K}\right]$ being the symbol bearing signal of the users and $\mathbf{P}$ is the precoding matrix with constraint $\mathrm{E}\left\{\left(\mathbf{P} \mathbf{P}^{\mathrm{H}}\right)\right\}=1$. $\mathbf{Q}$ is the downlink channel matrix defined as

$$
\mathbf{Q}=\sqrt{\mathbf{D}} \mathbf{H}
$$

where $\mathbf{H} \in \mathbb{C}^{M \times N}$ is the matrix of fast fading channel coefficients between the $M$ BS antennas and $N$ users. Every element of $\mathbf{H}$ is independent and identical distribution random variables with zero means and unit variance i.e. $\mathbb{C N}$ $(0,1), \mathbf{D}$ is a diagonal matrix which is written as

$$
[\mathbf{D}]_{k, k}=\beta_{k} .
$$

Assuming path loss and shadow fading, $\beta_{k}=R_{\mathrm{f}} /\left(R_{k} / R_{\mathrm{w}}\right)^{a} . R_{\mathrm{f}}$ is the shadow fading variable with the standard deviation $\sigma_{\text {shadow }}$ being a log-normal random variable. The distance from the $k^{\text {th }}$ users to the BS is $R_{k}$ and the path loss exponent is $a$. The maximum coverage distance of the cell is $R$ and there are no users within the minimum distance $R_{\mathrm{w}}$. The $k^{\text {th }}$ received signal vector is expressed as in (4). 


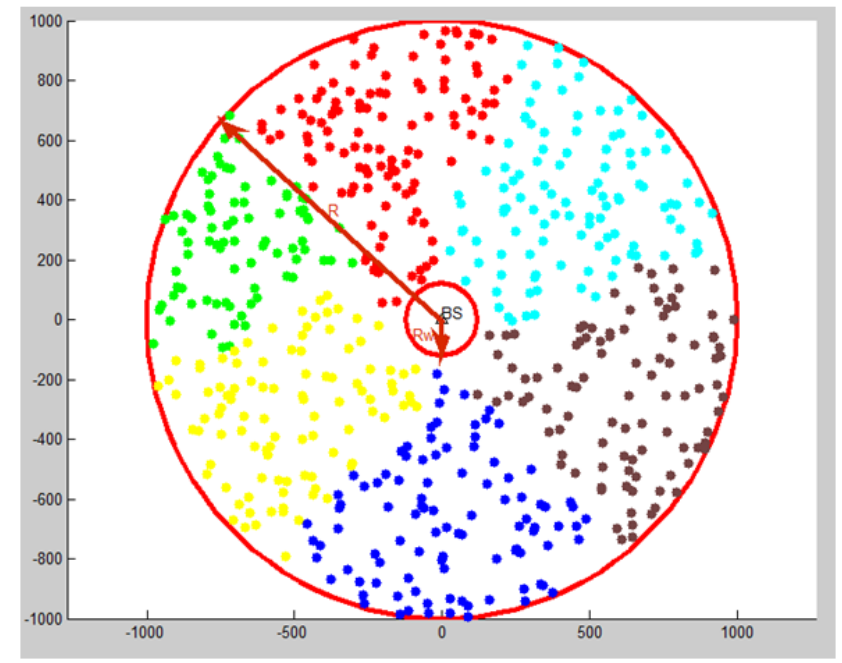

Fig. 1. Grouping of users in massive MIMO system.

$$
\mathbf{r}_{k}=\sqrt{\rho} \mathbf{q}_{k} \mathbf{P}_{k} s_{k}+\sqrt{\rho} \sum_{i=1, i \neq k}^{K} \mathbf{q}_{k} \mathbf{P}_{i} s_{i}+\mathbf{z}_{k}
$$

The $k^{\text {th }}$ row of $\mathbf{Q}$ is $\mathbf{q}_{k}, \mathbf{P}$ is $\mathbf{P}_{k}$ and for $\mathbf{z}$ is $\mathbf{z}_{k}$. Hence, using (3), equation (2) can be rewritten as

$$
\mathbf{q}=\sqrt{\beta_{k}} \mathbf{h}_{k} .
$$

The signal to interference plus noise ratio (SINR) of the $k^{\text {th }}$ user is

$$
\operatorname{SINR}=\frac{\left|\sqrt{\rho} \mathbf{q}_{k} \mathbf{P}_{k}\right|^{2}}{\sum_{i=1, i \neq k}^{K}\left|\sqrt{\rho} \mathbf{q}_{k} \mathbf{P}_{i}\right|^{2}+1} .
$$

Using (5) in (6), the above equation is rewritten as

$$
\begin{aligned}
& \operatorname{SINR}=\frac{\left|\sqrt{\rho \beta_{k}} \mathbf{h}_{k} \mathbf{P}_{k}\right|^{2}}{\sum_{i=1, i \neq k}^{K}\left|\sqrt{\rho \beta_{k}} \mathbf{h}_{k} \mathbf{P}_{i}\right|^{2}+1}, \\
& \operatorname{SINR}=\frac{\rho \beta_{k}\left|\mathbf{h}_{k} \mathbf{P}_{k}\right|^{2}}{\rho \beta_{k} \sum_{i=1, i \neq k}^{K}\left|\mathbf{h}_{k} \mathbf{P}_{i}\right|^{2}+1} .
\end{aligned}
$$

The system capacity rate $\mathbf{C}_{k}$ is for the $k^{\text {th }}$ user is

$$
C_{k}=\log _{2}(1+\operatorname{SINR}) \text {. }
$$

Equation (8) is finally expressed as

$$
C_{k}=\log _{2}\left(1+\frac{\rho \beta_{K}\left|\mathbf{h}_{k} \mathbf{P}_{k}\right|^{2}}{\rho \beta_{K} \sum_{i=1, i \neq k}^{K}\left|\mathbf{h}_{k} \mathbf{P}_{i}\right|^{2}+1}\right)
$$

\section{Description of Algorithms}

For the separation of users, we use $K$-means clustering algorithm. The users are separated into some predefined groups with the $K$-means clustering algorithm that is shown in Fig. 1. After the users are separated into groups used, SUS and RUS are used to determine the best users in each group. For the semi-orthogonality measurement, the angle $(\theta=0.1)$ among the users is considered. In intergroup and intra-group scenarios, both the SUS and RUS technique are used. In SUS algorithm the users are selected those are orthogonal to each other within the groups as well as in other groups. In the RUS method the users are selected randomly from each and every group. In both cases, antennas are selected based on the maximum gain of the channel. In both the algorithms, select 24 pairs of users and antennas from 6 groups and from each group select 4 numbers of users. All the algorithms are implemented and performed with MATLAB simulation software. After the selection and scheduling of antennas pair we use the ZF and MMSE precoding schemes to reduce the interference of the same group and other group users. The steps of SUS, RUS and AS algorithms are described below.

\section{Algorithm-1: Semi-orthogonal user selection (SUS)}

\section{SUS Steps;}

- Initialization: Number of BS antennas $M$; Number of users $N$;

- Iteration $i \leftarrow 1$;

- User set $\Theta \leftarrow\{1, \ldots, k, \ldots, N\}$;

- Selected user set $\mathfrak{I}_{K} \leftarrow \phi$

- To select first reference user, $\mathfrak{J}_{1}=\operatorname{argmax}_{K \in \Theta}\left\|\mathbf{h}_{k, \Gamma}\right\|^{2}$

- $\mathfrak{I}_{K} \leftarrow \mathfrak{I}_{K} \bigcup \mathfrak{I}_{1}$;

- While $i<K$

- For each $k$ in $\Theta$ do

- $\frac{\left|\mathbf{h}_{k}^{\perp} \mathbf{h}_{k-1}\right|}{\left\|\mathbf{h}_{k}\right\|\left\|\mathbf{h}_{k-1}\right\|} \leq \theta$

- end

- $\prod_{\mathrm{U}}=\operatorname{argmax}_{k \in \Theta}\left\|\mathbf{h}_{k, \Gamma}\right\|^{2}$

- $\mathfrak{I}_{K} \leftarrow \mathfrak{I}_{K} \cup \prod_{U} ; \quad \Theta \leftarrow \Theta \backslash \mathfrak{I}_{K}$

- Channel vector of selected user $\mathbf{h}_{K}=\mathbf{h}_{K, \Gamma}$

- $i=i+1$

- end

- $\mathfrak{I}_{\text {SUS }}=\mathfrak{I}_{K}$

\section{Algorithm-2: Random User Selection (RUS)}

\section{RUS Steps:}

- Initialization: Number of BS antennas $M$;

- Iteration $i \leftarrow 1$; Number of Users $N$;

- User set $\Theta \leftarrow\{1, \ldots, k, \ldots, N\}$;

- Selected user set $\mathfrak{I}_{K} \leftarrow \phi$

- While $i<K$

- Randomly select users $\Pi_{U}$ from $\Theta$

- $\mathfrak{I}_{K} \leftarrow \mathfrak{I}_{K} \cup \prod_{U} ; \quad \Theta \leftarrow \Theta \backslash \mathfrak{I}_{K}$

- Channel vector of selected user $\mathbf{h}_{K}=\mathbf{h}_{K, \Gamma}$

- $i=i+1$

- end

- $\mathfrak{I}_{\mathrm{RUS}}=\mathfrak{I}_{K}$ 


\section{Algorithm-3: Antenna Selection (AS)}

\section{AS Steps:}

- For each user in $\mathfrak{I}_{\mathrm{SUS} / \mathrm{RUS}}$, Channel vector of selected user $\mathbf{h}_{K}=\mathbf{h}_{K, \Gamma}$

- No. of BS antennas $M$; Iteration $j \leftarrow 1$;

- Base station set $\Gamma \leftarrow\{1, \ldots, m, \ldots, M\}$;

- Set of selected base station antennas $\mathfrak{I}_{K, N} \leftarrow \phi$

- While $j \leq N$

- $\Sigma_{A}=\operatorname{argmax}_{i \in \Gamma}\left\|\mathbf{h}_{k, i}\right\|^{2}$

- $\mathfrak{I}_{K, N} \leftarrow \mathfrak{I}_{K, N} \cup \Sigma_{A} ; \quad \Gamma \leftarrow \Gamma \backslash \mathfrak{I}_{K, N}$;

- Channel vector of selected user and antenna $\mathbf{h}_{K, N}=\mathbf{h}_{k, i}$

- $j \leftarrow j+1$

- end

- $\mathfrak{I}_{K, N}=\mathfrak{I}_{\text {SUS/RUS - AS }}$

\subsection{The Complexity of the SUS Algorithm}

In the SUS algorithm the count of flops begins from iteration $n=1$ to $N$. In every iteration, a newly selected semi-orthogonal user is added to the set containing earlier chosen users. In each iteration, new users which are semiorthogonal to the users in the set are found and from which the one with the largest channel vector norm is kept and added to the set, with $(n N)^{2} M$ flops. The algorithm terminates when the pre-definite number of semi-orthogonal users are selected. The final expression of $\varphi$ is as shown below.

$$
\begin{aligned}
& \varphi \approx \frac{1}{G}\left\{4 N M-\sum_{n=1}^{M}\left[\begin{array}{l}
N+\{M(16 n-12)-2 n+2\}+ \\
(12 M+1)(N-1)+N\}
\end{array}\right]\right\} \\
& \left(4 N M-N+N M+\frac{16 M^{2}(M+1)}{2}-\right) \\
& \approx \frac{1}{G}\left\{12 M^{2}-\frac{2 M(M+1)}{2}+2 M+\right. \\
& \left(12 M^{2}+M\right)(N-1)+N M \\
& \approx \frac{1}{G}\left\{\begin{array}{l}
6 N M-N+8 M^{3}+8 M^{2}-12 M^{2}-M^{2}-M+ \\
2 M+12 N M^{2}-12 M^{2}+N M-M
\end{array}\right\}, \\
& \varphi \approx \frac{1}{G}\left\{\mathrm{O}\left(M^{2} N\right)\right\} .
\end{aligned}
$$

Hence the complexity of the SUS algorithm is $1 / G\left\{O\left(N M^{2}\right)\right\}$.

\begin{tabular}{|c|c|}
\hline Reference & Computational Complexity \\
\hline Modified SUS algorithm & $\approx \frac{1}{G}\left\{O\left(M^{2} N\right)\right\}$ \\
\hline Reference [23] & $\approx O\left(N^{3}\right)$ \\
\hline Reference [24] & $\approx O\left(\frac{K}{3} \widehat{K}^{4} N^{3}\right)$ \\
\hline
\end{tabular}

Tab. 1. SUS algorithm's computation complexity comparison.

\begin{tabular}{|c|c|}
\hline Parameters & value \\
\hline$N$ & 600 \\
\hline downlink power $(\rho)$ & $8 \mathrm{~dB}$ \\
\hline Path loss exponent $(a)$ & 3.8 \\
\hline$\theta$ & 0.1 \\
\hline The radius of the coverage area $(R)$ & $1000 \mathrm{~m}$ \\
\hline Weak signaling zone $\left(R_{\mathrm{w}}\right)$ & $120 \mathrm{~m}$ \\
\hline User-selected from each from $(s)$ & 4 \\
\hline Number of groups & 6 \\
\hline Total number of selected users from different groups $(u)$ & 24 \\
\hline shadow fading variable $\left(R_{\mathrm{f}}\right)$ & $8 \mathrm{~dB}$ \\
\hline
\end{tabular}

Tab. 2. Simulation component value.

In RUS algorithm required only one iteration to select a pre-define number of users, so its flop count is 1 and complexity $O(1)$.

\section{Results and Discussion}

For exploring the performance of semi-orthogonal user selection and antenna scheduling (SUS-AS) and random user selection and antenna scheduling (RUS-AS) algorithms with ZF and MMSE precoding, we implemented the algorithms with the MATLAB simulation of the massive MIMO system varying the number of BS antennas. Throughout the simulation, we consider different parameters with values as shown in Tab. 2. The simulation is performed using MATLAB software.

Figure 2 shows the average sum-rate versus a number of BS antennas $M$ for two proposed scenarios in a massive MIMO network with two modified proposed algorithms with ZF and MMSE precoding. And $s=4$ numbers of users are selected and scheduled from each group and total $u=24$ numbers of users are selected from each model with scheduling algorithm SUS-AS and RUS-AS. The simulation results as shown in Fig. 2 show that the average system data-rate increases non-linearly with increasing $M$ for both the scenario. One of the two proposed scenarios in intragroup with SUS-AS with ZF precoding has the maximum data-rate whereas inter-group has the minimum. At smaller $M$ the difference of data-rate between scenarios is very small and at higher $M$ the difference is high.

In Fig. 3, we have plotted the performance of each proposed scenario separately with all the algorithms and precoding schemes keeping $N$ and $S N R$ the same. It is clear from Fig. 3 that the average data-rate increases almost linearly with $M$. Moreover, in Fig. 3 it is explored that the average data-rate is higher with all the algorithms and $\mathrm{ZF}$ precoding than MMSE precoding for both the scenarios. However, the average data-rate difference is less at lower $M$ and higher at higher values of $M$ in all the scenarios with all the algorithms and for both the precoding schemes.

We observe from Fig. 4 that the SUS-AS algorithm offered the highest average system data-rate than the RUS- 
AS algorithm. Also, the trend of an increase of average data-rate with a variation of BS antennas $M$ is almost similar in both the algorithms with ZF and MMSE precoding
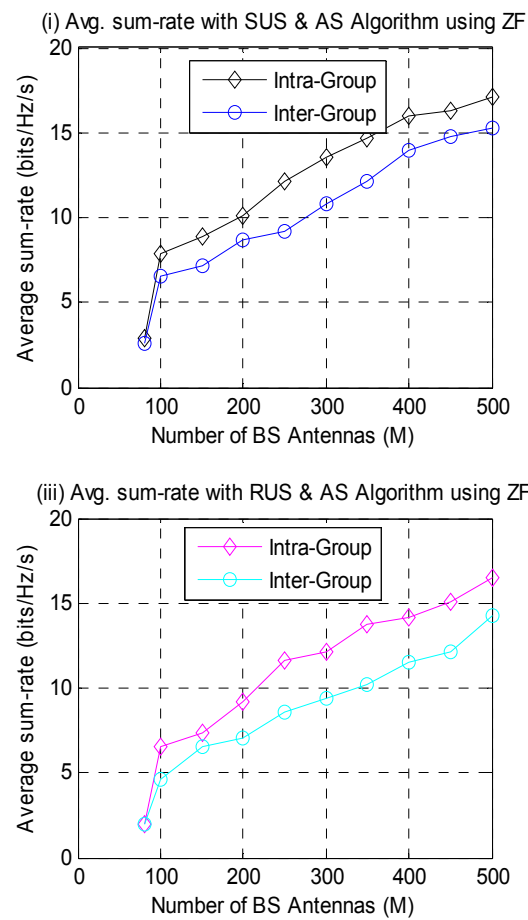

schemes for both the scenarios. However, the average sumrate difference is very small for both the scenarios with $\mathrm{ZF}$ and MMSE precoding.
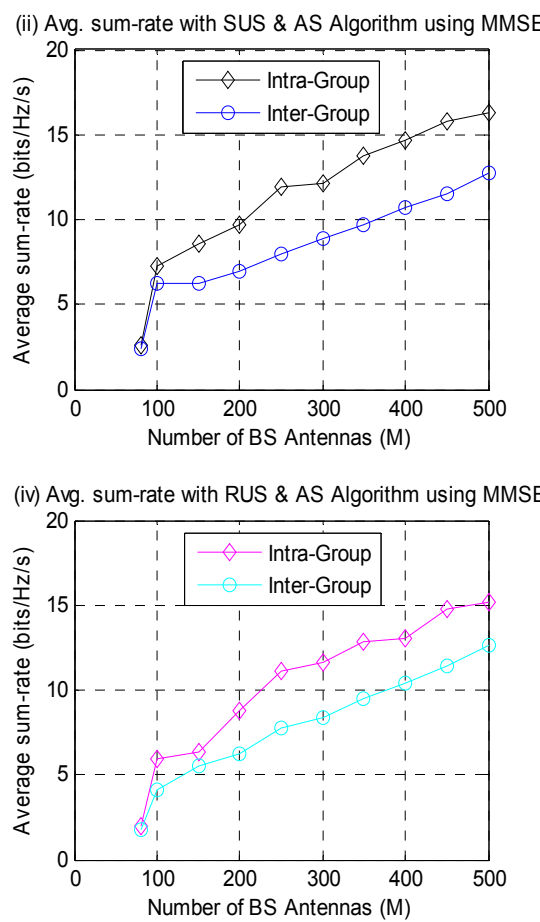

Fig. 2. Average data-rate versus number of BS antennas $M$ for different scenarios in massive MIMO network with (i) SUS-AS algorithm with ZF, (ii) SUS-AS algorithm with MMSE, (iii) RUS-AS algorithm with ZF, (iv) RUS-AS algorithm with MMSE.
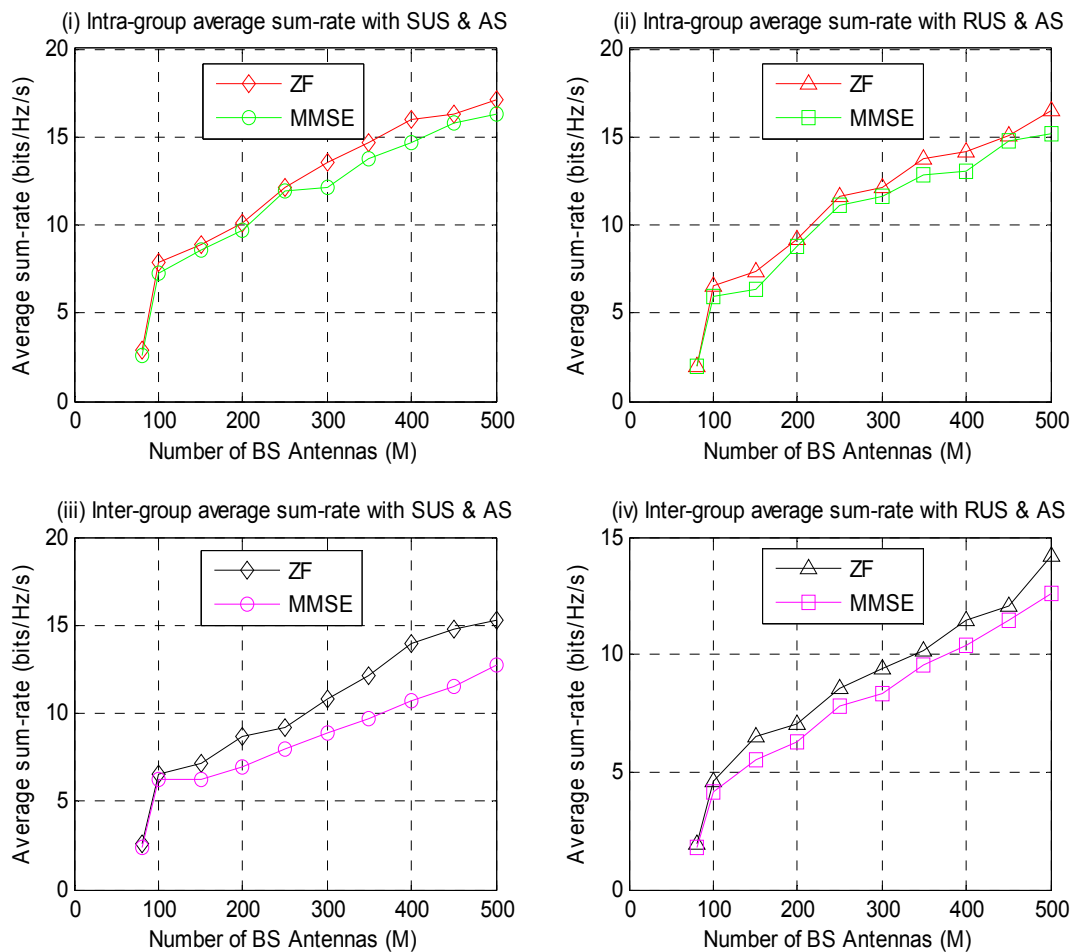

Fig. 3. Average data-rate versus the number of base station antennas $M$ for ZF and MMSE in (i) intra-group scenario with SUS-AS algorithm, (ii) intra-group scenario with RUS-AS algorithm, (iii) inter-group scenario with SUS-AS algorithm, (iv) intra-group scenario with RUS-AS algorithm. 

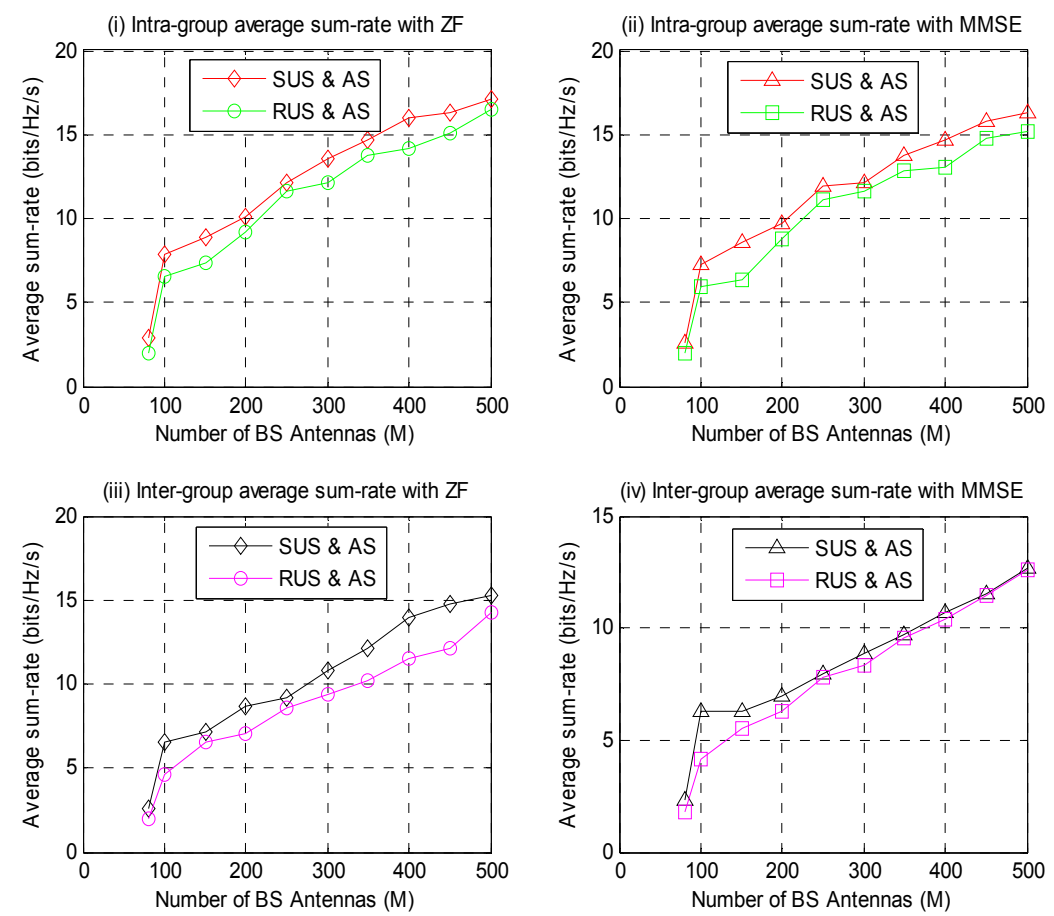

Fig. 4. Average data-rate versus the number of BS antennas $M$ for SUS-AS, and RUS-AS algorithms in (i) intra-group scenario with ZF, (ii) intra-group scenario with MMSE, (iii) inter-group scenario with ZF, (iv) intra-group scenario with MMSE.

\section{Conclusion}

We explore the simulation results for the performance of intra-group and inter-group massive MIMO system, considering perfect CSI and both SSF and LSF with ZF and MMSE precoding, with variation in the number of BS antennas. The evaluation of performance is studied using two user scheduling techniques such as intra-group and inter-group SUS; and intra-group and inter-group RUS. The antennas are scheduled based on the maximum gain of the channel in both cases. The users are distributed uniformly in the geographical area of radius 1000 meters. The results show that the average system data-rate increases non-linearly with a number of BS antennas irrespective of scheduling technique, SUS-AS being the highest with ZF and in the intra-grouping scenario, whereas RUS-AS being the lowest with MMSE and in inter-group. In this study, we have also measured the cost of system computational complexity in both the scenarios for the SUS-AS scheduling technique which is shown in Tab. 1 which also shows that our modified proposed SUS-AS algorithm has lower system computational cost. Moreover, one of the major advantages of our group-based SUS-AS algorithm is that when the number of groups in the massive MIMO system increased, the computational costs also decreased; that is observed in Tab. 1.

\section{Acknowledgments}

The authors thank to digital India corporation for financially supported this work under Visvesvaraya Ph.D. scheme (Grant no.: PhD-MLA-4(96)/2015-2016).

\section{References}

[1] LARSSON, E. G., EDFORS, O., TUFVESSON, F., et al. Massive MIMO for a next-generation wireless system. IEEE Communications Magazine, 2014, vol. 52, no. 2, p. 186-195. DOI: 10.1109/MCOM.2014.6736761

[2] SHEIKH, T. A., BORA, J., HUSSAIN, M. A. Combined user and antenna selection in massive MIMO using precoding technique. International Journal of Sensors, Wireless Communications, and Control, 2019, vol. 9, no. 2, p. 214-223. DOI: $10.2174 / 2210327908666181112144939$

[3] SHEIKH, T. A., BORA, J., HUSSAIN, M. A. A survey of antenna and user scheduling techniques for massive MIMO-5G wireless system. In 2017 International Conference on Current Trends in Computer, Electrical, Electronics and Communication (CTCEEC). Mysore (India), 2017, p. 578-583. DOI: 10.1109/CTCEEC.2017.8455177

[4] RUSEK, F., PERSSON, D., LAU, B. K., et al. Scaling up MIMO: Opportunities and challenges with very large arrays. IEEE Signal Processing Magazine, 2013, vol.30, no. 1, p. 40-60. DOI: 10.1109/MSP.2011.2178495

[5] SHEPARD, C., YU, H., ANAND, N., et al. Argos: Practical many-antenna base stations. In Proceedings of the 18th Annual International Conference on Mobile Computing and Networking (MOBICOM). Istanbul (Turkey), 2012, p. 53-64. DOI: $10.1145 / 2348543.2348553$

[6] SHEPARD, C., YU, H., ZHONG, L. ArgosV2: A flexible manyantenna research platform. In Proceedings of the 19th Annual International Conference on Mobile Computing and Networking (MOBICOM). Miami (FL, USA), 2013, p. 163-165. DOI: $10.1145 / 2500423.2505302$

[7] SHEIKH, T. A., BORA, J., HUSSAIN, M. A. Capacity maximizing in massive MIMO with linear precoding for SSF and LSF channel with perfect CSI. Digital Communications and Networks, 2019, p. 1-8 (Article in press). DOI: 10.1016/j.dcan.2019.08.002 
[8] SHEIKH, T. A., BORA J., HUSSAIN, M. A. Sum-rate performance of massive MIMO systems in highly scattering channel with semi-orthogonal and random user selections. Radioelectronics and Communications Systems, 2018, vol. 61, no. 12 , p. 547-555. DOI: $10.3103 / \mathrm{S} 0735272718120026$

[9] HOYDIS, J., HOSSEINI, K., TEN BRINK, S., et al. Making smart use of excess antennas: Massive MIMO, small cells, and TDD. Bell Labs Technical Journal, 2013, vol. 18, no. 2, p. 5-21. DOI: 10.1002/bltj.21602

[10] SHEIKH, T. A., BORA, J., HUSSAIN, M. A. Performance analysis of massive multi-input and multi-output with imperfect channel state information. Traitement du Signal, 2019, vol. 36, no. 4 , p. $361-368$. DOI: $10.18280 /$ ts.360409

[11] ADHIKARY, A., NAM, J., AHN, J. Y., et al. Joint spatial division and multiplexing: The large-scale array regime. IEEE Transactions on Information Theory, 2013, vol. 59, no. 10, p. 6441-6463. DOI: 10.1109/TIT.2013.2269476

[12] XU, Y., YUE, G., PRASAD, N., et al. User grouping and scheduling for large scale MIMO systems with two-stage precoding. In Proceedings of the IEEE International Conference on Communications (ICC). Sydney (Australia), 2014, p. 5208-5213. DOI: 10.1109/ICC.2014.6884146

[13] SUN, X., GAO, X., LI, G. Y., et al. Agglomerative user clustering and cluster scheduling for FDD massive MIMO systems. IEEE Access, 2019, vol. 7, p. 86522-86533. DOI: 10.1109/ACCESS.2019.2923246

[14] XU, Y., YUE, G., MAO, S. User grouping for massive MIMO in FDD systems: New design methods and analysis. IEEE Access, 2014, vol. 2, p. 947-959. DOI: 10.1109/ACCESS.2014.2353297

[15] HAJRI, S. E., ASSAAD, M., CAIRE, G. Scheduling in massive MIMO: User clustering and pilot assignment. In 2016 54th Annual Allerton Conference on Communication, Control, and Computing (Allerton). Monticello (IL, USA), 2016, p. 107-114. DOI: 10.1109/ALLERTON.2016.7852217

[16] WU, X., MA, Z., WANG, Y. Joint user grouping and resource allocation for multi-user dual-layer beamforming in LTE-A. IEEE Communications Letters, 2015, vol. 19, no. 10, p. 1822-1825. DOI: 10.1109/LCOMM.2015.2458861

[17] NAM, J., ADHIKARY, A., AHN, J. Y., et al. Joint spatial division and multiplexing: Opportunistic beamforming, user grouping, and simplified downlink scheduling. IEEE Journal of Selected Topics in Signal Processing, 2014, vol. 8, no. 5, p. 876-890. DOI: 10.1109/JSTSP.2014.2313808

[18] SONG, A., YANG, Q., CHEN, W. N., et al. A random-based dynamic grouping strategy for large scale multi-objective optimization. In 2016 IEEE Congress on Evolutionary Computation (CEC). Vancouver (BC, Canada), 2016, p. 468-475. DOI: $10.1109 /$ CEC.2016.7743831

[19] KUERBIS, M., BALASUBRAMANYA, N. M., LAMPE, L., et al. User scheduling in massive MIMO systems with a large number of devices. In 2017 IEEE 28th Annual International Symposium on Personal, Indoor, and Mobile Radio Communications (PIMRC). Montreal (QC, Canada), 2017, p. 1-6. DOI: 10.1109/PIMRC.2017.8292733

[20] WU, H., LIU, D., WU, W., et al. A low complexity two-stage user scheduling scheme for mm-wave massive MIMO hybrid beamforming systems. In The $3^{\text {rd }}$ IEEE International Conference on Computer and Communications (ICCC). Chengdu (China), 2017, p. 945-951. DOI: 10.1109/CompComm.2017.8322683

[21] CHEN, X., GONG, F., ZHANG, H., et al. Cooperative user scheduling in massive MIMO systems. IEEE Access, 2018, vol. 6, p. 21910-21923. DOI: 10.1109/ACCESS.2018.2828403

[22] NOVIANA SULISTYAWAN, V., PUDJI ASTUTI, R., FAHMI, A. Location-dependent user selection based on sum rate approximation in large system regime for massive MIMO. In The $1^{\text {st }}$ International Conference on Industrial, Electrical and
Electronics (ICIEE). 2018, vol. 218, p. 1-7. DOI: $10.1051 /$ matecconf $/ 201821803010$

[23] BENMIMOUNE, M., DRIOUCH, E., AJIB, W., et al. Joint transmit antenna selection and user scheduling for massive MIMO system. In IEEE Wireless Communication and Networking Conference (WCNC). New Orleans (LA, USA), 2015, p. 381-386. DOI: $10.1109 /$ WCNC.2015.7127500

[24] JIN, L., GU, X., HU, Z. Low-complexity scheduling strategy for wireless multiuser multiple-input multiple-output downlink system. IET Communications, 2011, vol. 5, no. 7, p. 990-995. DOI: 10.1049 /iet-com.2010.0358

\section{About the Authors ...}

Tasher Ali SHEIKH (corresponding author) received B.Tech. degree in Electronics and Communication Engineering from the Central Institute of Technology (CIT), Kokrajhar Assam, India, in 2013, M.Tech. in Mobile Communication and Computing from the National Institute of Technology (NIT), Arunachal Pradesh, India, in 2015, and Ph.D. from the North Eastern Regional Institute of Science and Technology (NERIST), Arunachal Pradesh, India in 2020. He is an IEEE Member, IETE and IEI Associate Member and Lifetime member of International Association of Engineers (IAENG), Member of American Association of Science and Technology (AASCIT). He has published research articles in international referred journals and conferences. His field of interest includes cell free massive MIMO, massive MIMO, microwave communication and wireless communication.

Joyatri BORA is currently working as an Assistant Professor in the Department of ECE at NERIST Deemed to be University, India. She did Ph.D. in Electronics \& Communication Engineering from NERIST, India in 2015. She received her M.Tech. degree in Electronics Design and Technology from Tezpur University, India in 2002 and B.Tech. degree in Electrical Engineering from Jorhat Engineering College, India in 1995. She has more than 10 years of experience in research and teaching field. She has published research articles in international referred journals and conferences. Her research interest includes wireless communication, massive MIMO, and bioelectronics.

Md. Anwar HUSSAIN received B.Sc. degree in Physics from Gauhati University, Assam, India, in 1981, B.Tech. and M.Tech. in Radio Physics \& Electronics from Calcutta University, West Bengal, India, in 1985 and 1987 respectively. Also, he received Ph.D. degree in Electronics and Communication Engineering from Jadavpur University, West Bengal, India, in 2002. He is currently a Professor in the Department of Electronics and Communication Engineering at NERIST, Arunachal Pradesh, India. He has more than 31 years of experience in research and teaching field. His research interests include massive MIMO, high data rate wireless communication \& networks, routing \& scheduling in multi-hop wireless networks, key distribution in sensor networks, multimedia data encryption \& security, mobile computing security, time-series data modeling and prediction, networks-on-chip. 